\title{
Breast Cancer Diagnosis, Treatment and Follow-Up During COVID-19 Pandemic
}

\author{
Atilla Soran (1), Adam Brufsky (1), Michael Gimbel (1), Emilia Diego (10) \\ Magee-Womens Hospital Comprehensive Breast Program, University of Pittsburgh Medical Center, Pittsburgh, PA, USA
}

Cite this article as: Soran A, Brufsky A, Gimbel M, Diego E. Breast Cancer Diagnosis, Treatment and Follow-Up During COVID-19 Pandemic. Eur J Breast Health 2020; 16(2): 86-88.

The COVID-19 Pandemic has not only changed our daily routines but also forced us to re-think the approach to cancer patients in these unprecedented times. Breast cancer is the most common cancer in women globally. It is unfortunate that there are subsets of those affected by COVID-19 who will experience more severe symptoms and are considered a vulnerable population: cancer patients fall into this category. Fortunately, developments in breast cancer diagnosis and treatment provide us with options such as neoadjuvant systemic therapy in the form of hormonal therapy, anti-HER2 or systemic chemotherapy and a variety of modifiable breast reconstruction strategies.

Based on the "CMS Adult Elective Surgery and Procedures Recommendations (1)" breast cancer is a Tier 2a, low risk cancer and similar to The U.S. Centers for Disease Control and Prevention, and The American College of Surgeons, the recommendation is to postpone surgery if possible or consider performing the procedure at an ambulatory surgery center.

It is obvious that we should minimize utilization of medical resources and supplies for non-urgent procedures; this approach helps us to maintain reserves that may be needed to care for COVID-19 affected patients, including personal protective equipment, ICU beds, cleaning supplies, and ventilators. We should also keep in mind that every hospital visits exposes patients and health care providers to the risk of contracting COVID-19. The American College of Surgeons (ACS) (2) has issued a statement and distributed to their members that "each hospital, health system, and surgeon should thoughtfully review all scheduled elective procedures with a plan to minimize, postpone, or cancel electively scheduled operations, endoscopies, or other invasive procedures until we have passed the predicted inflection point in the exposure graph and can be confident that our health care infrastructure can support a potentially rapid and overwhelming uptick in critical patient care needs."

Although medical centers must continue to offer care that cannot be post-poned for months for some non-COVID-19 related conditions (e.g. cancer), the benefits of this care should be balanced against the risk of COVID-19 exposure for patients and staff during this pandemic. For each breast cancer patient, the risk/benefit ratio should be considered; if the risk of postponing breast surgery (breast imaging as well) confers minimal to no harm (defined as a negative impact on survival), then the option of postponing the surgery until the COVID-19 pandemic is under better control should be discussed with patient.

Herein, we present the current approach to COVID-19 pandemic of the Magee-Breast Cancer Program, one of the busiest breast care centers in the US. The Magee Breast Cancer Program leaders (Surgery, Medical Oncology, Radiation Oncology, Plastic Surgery, Pathology and Genetics) came to a consensus and prepared a statement (updated every week) that may guide breast care professionals.

Acknowledgements: The Magee Breast Cancer Program Response to COVID-19 Pandemic table was prepared with the help of Drs. Margarita Zuley, Rohit Bhargava, Ronald Johnson, Sushil Beriwal, and Phuong Mai.

\section{References}

1. Available from: https://www.cms.gov/files/document/31820-cms-adult-elective-surgery-and-procedures-recommendations.pdf Created by: Sameer Siddiqui MD Version 3.15.20.

2. Available from: https://www.facs.org/about-acs/covid-19/information-for-surgeons/triage 
Table 1. Magee Breast Cancer Program Response to COVID-19 Pandemic

Breast Imaging 1. Screening and routine, non-urgent diagnostic imaging: all patients will be contacted and given the option to defer to a later date

2. Urgent diagnostic imaging and biopsies will proceed as usual

3. Seed placement (or wire) will be scheduled on same day as surgery to minimize hospital trips for patients

Pathology $\quad$ 1. Will work to keep reporting timeline for biopsies as usual

2. Receptor status for DCIS will be on a per-request basis

3. Surgical pathology reporting timelines may be delayed by 1 ог 2 more days longer than usual

4. Intraoperative frozen sections to be reported via phone call into OR instead of in-person pathology report to minimize use of PPEs

Surgery Surgical Consultations/Appointments

1. New patients scheduled for breast cancer consultations will be seen with an in-person visit to establish plan of care

2. New patients scheduled for non-cancer consultations will be contacted by scheduled provider or to offer a virtual visit or deferred in-person appointment

3. Mid chemotherapy visits should be contacted for a virtual visit

4. End chemotherapy visits can be done at the discretion of the provider with a preference for a video visit when possible

5. Post-operative visits will be seen as a virtual visit unless there is need for an in-person appointment (i.e., drain pull, incision and drainage)

6. Breast Cancer Surveillance/follow-up appointments will be contacted for the option to have virtual visit or postponement of appointment to later date

7. New patients for lymphedema will be contacted for the option to have a virtual visit or postponement of appointment to later date

8. Surveillance for lymphedema will be contacted for option to have a virtual visit or postponement of appointment to later date

Surgical

Procedures
1. Patients who cannot afford a delay in surgical intervention will be offered a procedure immediately

a. Those who have completed chemotherapy and have no other alternative medical therapies

b. Those who have T1a-bN0 (premenopausal) T1a-cN0 (postmenopausal) triple negative or ER negative, HER2 positive breast cancer

c. Those for whom systemic chemotherapy is not an option (elderly/frail triple negative or HER2 positive breast cancer)

2. Patients who can reasonably postpone their operation without compromising outcomes will be offered medical intervention while awaiting surgery

a. Hormone receptor positive breast cancers (including ER positive DCIS):

i. Tamoxifen $20 \mathrm{mg}$ PO Q day if premenopausal, with reassessment of situation every 2 weeks (for feasibility of surgical intervention)

ii. Anastrozole $1 \mathrm{mg}$ PO Q day if postmenopausal, with reassessment of situation every 2 weeks (for feasibility of surgical intervention)

b. Premeonopausal women with T1cN0 or greater triple negative breast cancers or ER negative, HER2 positive breast cancers:

i. Neoadjuvant systemic chemotherapy

c. Postmenopausal women with T2N0 triple negative breast cancers, discuss with medical oncology regarding merits of neoadjuvant systemic therapy or primary surgical intervention

d. Hormone receptor positive breast cancers who have completed chemotherapy and demonstrate an imaging response should be continued on systemic therapy with tamoxifen of anastrozole

e. High risk and benign surgeries

f. Risk-reducing surgeries

Medical Oncology 1. Surveillance and routine follow-up visits to be done via telemedicine, e-mail or phone (provider preference).

2. Infusions and monthly injections to proceed as usual. 


\section{Table 1. Magee Breast Cancer Program Response to COVID-19 Pandemic}

3. Metastatic breast cancer patient protocols to proceed as usual.

Plastic Surgery 1. In general, much of breast plastic surgery is not time dependent. Outcomes are identical or similar if performed several months after originally scheduled surgical date. These types or procedures include delayed breast recon, second stage breast recon, cosmetic surgery, breast reductions, and other non-cancer related issues.

2. Breast reconstruction as a part of breast cancer treatment has more nuanced issues that can impact how timing of surgery affects results. For the most part, performing delayed reconstruction several months after mastectomy results in similar results as performing it in the immediate setting, with some exceptions.

We are favoring delayed reconstruction after the Covid-19 crisis has abated unless there are extenuating circumstances that suggest clear benefit of immediate reconstruction over delayed. These will be assessed on a case-by-case basis. We are asking our referring physicians to use their best judgement in determining whether a patient has an extenuating circumstance necessitating immediate reconstruction. If they do, we will conduct a virtual appointment with these patients. If not, we request that they hold for a physical plastic surgery appointment until after the pandemic.

3. As much as possible, clinic appointments will be converted to virtual appointments. There will still be a need for physical appointments for recent surgical patients requiring TE inflations, drain removal, and assessment for potential complications.

Radiation 1. New consultations will be seen via telemedicine if needed.

Oncology

2. Timelines for treatment of most patients to remain the same.

3. Timelines for low risk, luminal patients may be delayed without compromise. Additionally, patients will be offered abbreviated, 1-week course instead of usual 3 weeks.

Genetics 1. Urgent consultations still seen in person (blood draw done same day)

2. Non-urgent consultations already on schedule offered telemedicine appointment or option to reschedule to later date (blood draw done at later date)

3. The service is currently only scheduling urgent patients

Research 1. Biospecimen lab. is closed.

2. Clinical trial accrual still open

Multidisciplinary Meetings and Teaching Conferences

Proceed as scheduled using video conference 